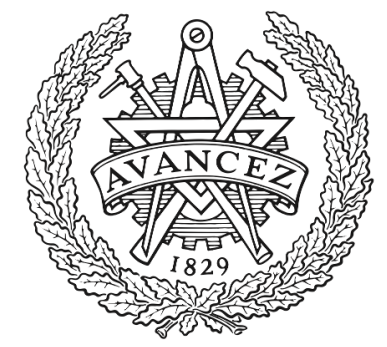

CHALMERS

UNIVERSITY OF TECHNOLOGY

\title{
The challenge of predicting spinnability: Investigating benefits of adding lignin to cellulose solutions in air-gap spinning
}

Downloaded from: https://research.chalmers.se, 2023-04-26 12:49 UTC

Citation for the original published paper (version of record):

Bengtsson, J., Jedvert, K., Köhnke, T. et al (2021). The challenge of predicting spinnability:

Investigating benefits of adding lignin to cellulose solutions in air-gap spinning. Journal of Applied Polymer Science, 138(26).

http://dx.doi.org/10.1002/app.50629

N.B. When citing this work, cite the original published paper. 


\title{
The challenge of predicting spinnability: Investigating benefits of adding lignin to cellulose solutions in air-gap spinning
}

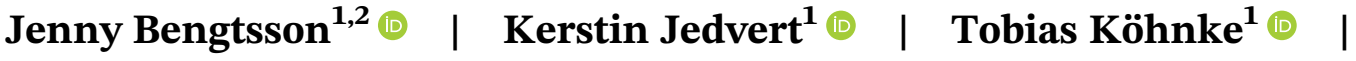 \\ Hans Theliander ${ }^{2}$ ()
}

${ }^{1}$ Fiber Development, RISE Research Institutes of Sweden, Mölndal, Sweden

${ }^{2}$ Division of Forest Products and Chemical Engineering, Department of Chemistry and Chemical Engineering, Chalmers University of Technology, Göteborg, Sweden

\section{Correspondence}

Jenny Bengtsson, Division of Forest Products and Chemical Engineering, Department of Chemistry and Chemical Engineering, Chalmers University of Technology, Kemigården 4, 412

96, Göteborg, Sweden.

Email: jenny.bengtsson@ri.se

Funding information

Energimyndigheten, Grant/Award

Number: 39573-2; Swedish Energy Agency

\begin{abstract}
In this study, the underlying mechanism for improved spinnability when mixing lignin and cellulose in solution was investigated. Co-processing of lignin and cellulose has previously been identified as a potential route for production of inexpensive and bio-based carbon fibers. The molecular order of cellulose contributes to the strength of the fibers and the high carbon content of lignin improves the yield during conversion to carbon fibers. The current work presents an additional benefit of combining lignin and cellulose; solutions that contain both lignin and cellulose could be air-gap spun at substantially higher draw ratios than pure cellulose solutions, that is, lignin improved the spinnability. Fibers were spun from solutions containing different ratios of lignin, from 0 to $70 \mathrm{wt} \%$, and the critical draw ratio was determined at various temperatures of solution. The observations were followed by characterization of the solutions with shear and elongational viscosity and surface tension, but none of these methods could explain the beneficial effect of lignin on the spinnability. However, by measuring the take-up force it was found that lignin seems to stabilize against diameter fluctuations during spinning, and plausible explanations are discussed.
\end{abstract}

\section{K E Y W O R D S}

cellulose and other wood products, extrusion, rheology

\section{1 | INTRODUCTION}

Carbon fiber reinforced composites are an attractive replacement of traditional construction materials, such as steel, as the high strength of carbon fibers enables an overall reduction in weight. ${ }^{1}$ A few examples, where the use of carbon fiber reinforced composites may lower the environmental impact, are electric windmills, ${ }^{2}$ concrete, ${ }^{3}$ and vehicles. ${ }^{4,5}$ Carbon fibers consist of more than $92 \%$ carbon and they are produced from polymeric precursor fibers which are converted into carbon fibers through thermal processes. ${ }^{6}$ However, the majority of the current commercial precursor fibers are produced from very expensive and fossil-based starting materials. Thus, less expensive, and preferably biobased raw materials for carbon fibers are sought for. Recent research has shown great promise in carbon fibers produced from a combination of lignin and cellulose. ${ }^{7,8}$ The advantage of using lignin and cellulose is that they are both bio-based and abundant materials, thus enabling a less expensive carbon fiber. ${ }^{9}$ Furthermore, in such carbon fibers, cellulose contributes with high strength and lignin with high carbon yield after the conversion into carbon fibers. ${ }^{10,11}$

As cellulose does not melt, lignin-cellulose precursor fibers must be produced by solution spinning. To 
perform solution spinning, the polymers must first be dissolved in a common solvent and ionic liquids (ILs) have been found to be a promising group of solvents. ${ }^{12-14}$ Dry-jet wet (air-gap) spinning, a type of solution spinning that includes extruding the solution via an air-gap into a coagulation bath, has proven to be a suitable method to manufacture lignin-cellulose fibers. $^{12,14}$

During air-gap spinning, the fibers are stretched by increasing the take-up velocity with respect to the extrusion velocity. The ratio of the take-up and extrusion velocity is known as the draw ratio. Particularly during air-gap spinning of cellulose solutions, a high draw ratio may improve the mechanical properties of the fibers ${ }^{15}$ since the solution is stretched in the airgap, and the polymers thus are oriented within the fiber before coagulation. The maximum achievable draw ratio before filament breakage, denoted critical draw ratio, is limited due to instabilities that may occur during spining. ${ }^{16}$ The different breach mechanisms during air-gap spinning of a lignin-cellulose solution were recently studied in our lab. The critical draw ratio was determined and optically analyzed at different temperatures, and two dominating mechanisms were found. ${ }^{17}$ At colder temperatures, in this case below $60^{\circ} \mathrm{C}$, the filaments were proposed to undergo a cohesive breach as the stored elastic stress in the filament exceeded the breaking stress, and at warmer temperatures, above $60^{\circ} \mathrm{C}$, the break-up was found to be caused by draw resonance, which induce diameter fluctuations in the filament.

Furthermore, in our previous work, it was noted that the addition of lignin to an IL-cellulose solution facilitated higher draw ratios during spinning. ${ }^{10}$ However, this increase was not quantified and it remained unexplored as to the reasons why the lignin-cellulose solutions could be stretched a lot more compared to neat cellulose solutions. This lack of understanding stresses the need to study the solutions in more detail. Consequently, the present work investigates the effect of addition of lignin to a cellulose solution on the properties of the solution, linked to its spinning performance. The critical draw ratio during air-gap spinning was mapped for several solutions, combinations of cellulose and lignin dissolved in EMIMAc, and temperatures. The solutions were evaluated with shear and extensional rheology, as well as surface tension. The take-up force during spinning was also measured with increased draw ratio. The information obtained contributes to better understanding of the air-gap spinning process in general and is thus useful for designing stable spinning processes.

\section{$2 \mid$ EXPERIMENTAL}

\section{1 | Materials}

Softwood Kraft lignin was received from Bäckhammar Pilot Plant (Bäckhammar, Sweden) where it was isolated through the LignoBoost method using industrial black liquor. ${ }^{18}$ Softwood dissolving grade pulp, Buckeye v67, was purchased from Georgia Pacific (Atlanta, GA). The intrinsic viscosity was determined to $465 \mathrm{ml} / \mathrm{g}$ according to ISO 5351. The IL solvent 1-ethyl-3-methylimidazolium acetate (EMIMAc, Aldrich 95\%) was used as received.

\section{2 | Dissolution}

Before use, the lignin was passed through a $0.5 \mathrm{~mm}$ sieve and dried overnight at $60^{\circ} \mathrm{C}$ in 100 mbar. Pulp sheets were chopped, ground, and dried overnight at $40^{\circ} \mathrm{C}$ prior to dissolution, the pulp is hereafter referred to as cellulose. Lignin and cellulose were combined at three different ratios $(30 \%$, $50 \%, 70 \%$ lignin). Two neat cellulose samples were also prepared for reference; with the same cellulose concentration as the lignin containing solutions ( $8 \mathrm{wt} \%$ ) and with the same total solids concentration as the $30 \%$ lignin solution (11.4 wt $\%$ ), see Table 1. Lignin and cellulose were dissolved together in EMIMAc at $70^{\circ} \mathrm{C}$ for $1 \mathrm{~h}$ in a closed reactor with overhead stirring at $30 \mathrm{rpm}$. Successful dissolution was confirmed by no visible particle in the solutions in optical light microscopy equipped with crossed polarizers. Deaeration was done at $60^{\circ} \mathrm{C}$ below $10 \mathrm{kPa}$ pressure for at least $5 \mathrm{~h}$.

\section{3 | Rheology of solutions}

The solutions were analyzed through oscillating rheometry on a CS Rheometer (Bohlin Instruments, Cirencester, UK) equipped with a cone/plate-geometry $\left(25 \mathrm{~mm} / 5^{\circ}\right)$ to obtain the temperature-viscosity relation for each spin dope. Master curves for the complex viscosity and shear moduli were created according to time-temperature-superposition method described by Metzger ${ }^{19}$ and only the horizontal shift factor, $a_{\mathrm{T}}$, was used. The zero shear viscosity was calculated by fitting the master curve of the complex viscosity to the Cross Model, after $\eta_{\infty}$ was set to zero, (using Matlab Curvefitting tool) according to Equation (1). ${ }^{20}$

$$
\eta=\frac{\eta_{0}}{\left(1+K \dot{\gamma}^{(1-n)}\right)}
$$

where $\eta$ is the complex viscosity, $\eta_{0}$ is the zero-shear viscosity, $K$ is a time constant, $\gamma$ is the shear rate, and $n$ is a 
TABLE 1 Composition of prepared solutions

\begin{tabular}{lllll}
\hline Sample name & Cellulose, wt\% & Lignin, wt\% & Lignin ratio, \% & Total solids content, wt\% \\
\hline L0C8 & 8 & 0 & 0 & 8 \\
\hline L30C8 & 8 & 3.4 & 30 & 11.4 \\
\hline L0C11.4 & 11.4 & 0 & 0 & 11.4 \\
\hline L50C8 & 8 & 8 & 50 & 16 \\
\hline L70C8 & 8 & 18.7 & 70 & 26.7 \\
\hline
\end{tabular}

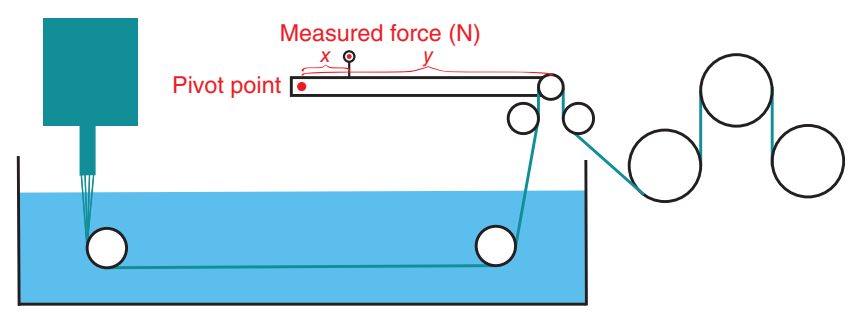

F I G U RE 1 Spinning set-up with force measurement. Filaments passed over a godet roll attached to a lever before collected at the take-up rolls. A tensiometer was attached on the lever where indicated (measured force) [Color figure can be viewed at wileyonlinelibrary.com]

power-law exponent. Since small strains, 0.01, were applied and thereby within the linear viscoelastic region, the viscosity is expected to follow the Cox-Merz rule. ${ }^{21}$

\section{4 | Spinning of filaments}

The solution was spun in a bench-scale spinning equipment, which consists of a piston pump, spin bath, and take-up rolls according to Figure 1. Six different temperatures of the dope were used, from 30 to $105^{\circ} \mathrm{C}$, evenly distributed with $15^{\circ} \mathrm{C}$ increment, as well as one additional spinning at $68^{\circ} \mathrm{C}$ only for L50C8 to locate the maximum critical draw ratio in some detail. Solutions were tempered for $45 \mathrm{~min}$ before spinning at each temperature. Extrusion was performed through a multi-filament die (4 holes, $150 \mu \mathrm{m}$ capillary diameter, L/D 3) over an airgap of $10 \mathrm{~mm}$ into a spin-bath of deionized water at $5-10^{\circ} \mathrm{C}$. Calculations showed that, at the conditions in the coagulation bath, the thermal diffusivity is substantially faster than the mass transport, therefore, it can be assumed that the filaments, regardless of the temperature of the spinning solution, obtain the temperature of the coagulation bath virtually instantaneously. Extrusion velocity was fixed at $4 \mathrm{~m} / \mathrm{min}$. Take-up speed $\left(v_{\mathrm{t}}\right)$ during spinning was increased incrementally, with stable spinning conditions in between increments, until filament breach. The speed at which the first and last filaments broke was noted. When the first filament broke, it may be due to imperfections in solution, such as air bubbles or other inhomogeneities. The breakage of the last filament was likely a critical breach, limited by the properties of solution. This procedure was repeated five times for each temperature. The fibers were subsequently washed in water for $24 \mathrm{~h}$ and treated with fabric softener (Neutral®, Unilever, Copenhagen, Denmark) for improved handling before drying at constant length at $80^{\circ} \mathrm{C}$ for $45 \mathrm{~min}$.

\section{5 | Measurement of take-up force during spinning}

The take-up force was measured according to Figure 1, a photograph of the equipment is available in the supporting information, Figure S3. To achieve reliable force measurements, a die with 33 holes was used, otherwise the spinning parameters were the same as described above. The take-up force measurement was aborted at a draw ratio lower than the draw ratio where some filaments started to break.

The take-up force was calculated from the measured force according to:

$$
F_{\mathrm{t}}=\frac{F_{\mathrm{m}}}{2 l}
$$

In Equation (2), $F_{\mathrm{t}}$ is the take-up force, $F_{\mathrm{m}}$ the measured force and $l$ is the leverage ( $y / x$ in Figure 1).

\subsection{Calculation of stress in filament}

The take-up force $F_{\mathrm{t}}$ is a result of the resistance of stretching the solution in the air-gap and the friction from pulling the filaments through the coagulation bath. To estimate the frictional force, $F_{\mathrm{f}}$, spinning was performed with four different coagulation bath lengths $(0.24$, $0.3,0.4$, and $0.5 \mathrm{~m}$ ) and with four different draw ratios $(1,4,8$, and 10$)$ for the solution $\mathrm{L} 50 \mathrm{C} 8$ at $60^{\circ} \mathrm{C}$. The particular solution and temperature were chosen to enable a high number of possible draw ratios. The frictional force 
arises from the shear at the filament surface when pulled through the coagulation bath and may be described according to Equation (3). ${ }^{22}$

$$
\begin{gathered}
F_{\mathrm{f}}=2 \pi R_{\mathrm{L}} L_{\mathrm{c}} \frac{1}{2} \rho v_{\mathrm{L}}^{2} C_{\mathrm{f}} \\
C_{\mathrm{f}}=\mathrm{A}(\mathrm{Re})^{-x}
\end{gathered}
$$

In Equations (3) and (4), $R_{L}$ is the total filament tow radius, $L_{c}$ is the coagulation bath length, $\rho$ is the density of water (coagulation bath liquid), and $v_{L}$ is the filament velocity. $C_{\mathrm{f}}$ is the skin-friction coefficient that may be expressed by Equation (4) where Re is the Reynold's number. The calculation of the constants $A$ and $x$ was performed by fitting the data of the take-up force from the spinning measurements using coagulation baths of different lengths and may be followed in detail Section S6. It was assumed that all stretching take place in the airgap and thereby the filament has a constant velocity through the coagulation bath. Furthermore, the filaments were assumed to be cylindrical. Finally, an expression for the frictional force as a function of draw ratio was obtained, and regard was taken to the different filament radius for different solutions due to their different solid content.

At the end of the air-gap, the resisting force will be a sum of several forces ${ }^{22}$ :

$$
F_{\mathrm{r}}=F_{\text {rheo }}+F_{\mathrm{s}}+F_{\mathrm{i}}-F_{\mathrm{g}}
$$

In Equation (5), $F_{\mathrm{r}}$ is the total resisting force, $F_{\text {rheo }}$ is the viscoelastic force, depending on the viscosity and the elasticity of the fluid, $F_{\mathrm{S}}$ arises from the surface tension, $F_{\mathrm{i}}$ is the inertia it has to overcome and $F_{\mathrm{g}}$ is the pushing from gravity due to vertical spinning. Gravity will only matter in the air gap since the coagulation bath (water) and the filament have similar density. ${ }^{23}$ If using approximations made by Ziabicki ${ }^{22}$ it is possible to conclude that surface tension, inertia and gravity may be neglected, for an in depth discussion please refer to the supporting information S1. Thus, the resisting force is approximated to be equal to the rheological force, according to Equation (3), and can thereby be used to calculate the stress in the extruded filament.

$$
\sigma=\frac{F_{\mathrm{r}}}{A}=\frac{F_{\mathrm{r}} D_{\mathrm{R}}}{\pi R_{\mathrm{e}}^{2} n}
$$

In Equation (6), $\sigma$ is the stress in the filament, $R_{\mathrm{e}}$ is the radius of the extruded filament, $D_{\mathrm{R}}$ is the draw ratio, and $n$ is the number of filaments.

\section{7 | Density and surface tension}

The density of the solutions was measured with a Hepycnometer AccuPycII 1340 (Micromeritics Instrument, Norcross) at room temperature, and the temperature dependence was taken as an average of the relation found for EMIMAc by Sescousse et al. ${ }^{24}$ and Schuermann et al. ${ }^{25}: \rho_{\text {EMIMAc }}=-0.00055 \mathrm{~T}+1.1209$, which correlated very well with the value obtained at RT $\left(22^{\circ} \mathrm{C}\right)$. From the results of Schuermann et al. ${ }^{25}$ it was assumed that the density of the cellulose solutions decreases with temperature with the same coefficient as EMIMAc. All values of measured density are available in Table S2.

Surface tension measurements were performed using the pendant drop method with an optical drop contour analysis, OCA 40 Micro (DataPhysics Instruments, Filderstadt, Germany). The temperature was controlled with a temperature chamber in combination with a temperature-controlled dosing system. The syringe had a diameter of $1.83 \mathrm{~mm}$ and the drop contour was determined through the software SCA202 V.5.0.32 build 5032 (DataPhysics Instruments, Filderstadt, Germany) Temperature was set to either 45,60 , or $75^{\circ} \mathrm{C}$, apart from for L0C 11.4 which was not analyzed at $45^{\circ} \mathrm{C}$ due to too high viscosity. Another effect resulting from the high viscosity of the solutions is the time needed for drop formation. The drops were recorded and the final shape of the drop, and thereby the final surface tension, was not received until after 10-20 min, depending on the temperature. The measured surface tension increased up to a plateau value during the formation of the drop. The value of surface tension was taken as the average plateau value, an example of the measurement is visible in Figure S2. To our knowledge, the time for drop formation needed for correct surface tension measurement of viscous cellulose ionic-liquid solutions has not previously been reported.

The long measurement time might be an issue with EMIMAc since it is hygroscopic and adsorbed water on the EMIMAc surface might increase the measured surface tension. However, for all measured temperatures the difference between the initial and final surface tension (measured after $20 \mathrm{~min}$ ) was rather small, in the range of $2 \mathrm{mN} / \mathrm{m}$ (from an initial value of $45.7-47.5 \mathrm{mN} / \mathrm{m}$ ). The increase of surface tension due to uptake of water in EMIMAc was thereby considered to be limited. The value of surface tension of EMIMAc was considered as the average value from a 20 min measurement, and the surface tension decreased slightly with temperature, as was expected. $^{26}$ 


\section{8 | Capillary thinning measurements, CaBER}

The extensional behavior of the solutions was analyzed with Capillary Break-up Elongational Rheometry (Haake CaBER 1, Thermo Haake, Karlsruhe, Germany). The measurement set-up compromises of two circular plates with a diameter of $4 \mathrm{~mm}$ and an initial separation of $2 \mathrm{~mm}$. For successful sample loading the two plates were heated to $75^{\circ} \mathrm{C}$. The plates were separated at time $t=-50 \mathrm{~ms}$, with a linear velocity profile until time $t=0 \mathrm{~s}$, to a final gap of $6 \mathrm{~mm}$. The diameter of liquid thread at the midplane between the two plates was monitored with a laser micrometer at a frequency of $10 \mathrm{~Hz}$ and with a detection limit of $10 \mu \mathrm{m}$.

\section{9 | Filament characterization}

Tensile testing (Vibroskop/Vibrodyn, Lenzing Instruments, Austria) was performed on conditioned fibers at $20 \pm 2{ }^{\circ} \mathrm{C}$ and $65 \pm 3 \% \mathrm{RH}$ with an extension rate of $20 \mathrm{~mm} / \mathrm{min}$ and a gauge length of $20 \mathrm{~mm}$. Conversion to SI units $(\mathrm{Pa})$ was done based on a fiber density of $1390 \mathrm{~kg} / \mathrm{m}^{3}$, obtained by a He-pycnometer AccuPycII 1340 at room temperature. Images of the surface of the filaments were taken with a scanning electron microscope (SEM) from JEOL, model JSM-7800F. The filaments were coated with $1.5 \mathrm{~nm}$ of platinum. Secondary electrons images were acquired using an accelerating voltage of $5 \mathrm{kV}$ and a working distance of $4 \mathrm{~mm}$.

\section{3 | RESULTS}

\section{1 | Spinning of filaments, critical draw ratio}

Figure 2 provides critical draw ratios for each solution at different temperatures. It can be clearly observed that a higher lignin ratio in the solution enabled a higher critical draw ratio, which is a strong indication of a better spinnability for these solutions. This increase in critical draw ratio may be explained by the higher solid content of the solution, as it may enhance its breaking stress. On the other hand, the L30C8 and L0C11.4 solutions have the same solid content, but L30C8, which contains lignin, attained a nearly twice as high maximum critical draw ratio. Accordingly, the addition of lignin to a cellulose solution has a beneficial effect on the maximum draw ratio.

Figure 3 shows that solutions also containing lignin achieved their maximum draw ratios at lower viscosities



F I G URE 2 Critical draw ratio $\left(v_{\mathrm{t}} / v_{\mathrm{e}}\right)$ for each solution at selected temperatures. The shadowed areas are added to help guide the eye to the maximum critical draw ratio of each solution. $N=5$ [Color figure can be viewed at wileyonlinelibrary.com]

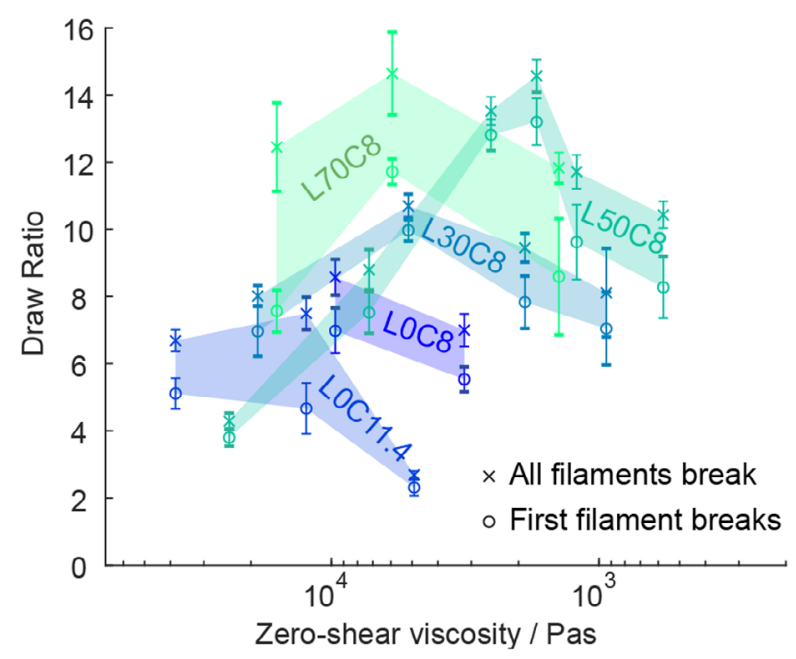

F I G URE 3 Critical draw ratio $\left(v_{\mathrm{t}} / v_{\mathrm{e}}\right)$ for all solutions versus zero-shear viscosity of the solution at the different temperatures in Figure 2, note the declining viscosity on the $x$-axis. The shadowed areas are added to help guide the eye to the maximum critical draw ratio of each solution. $N=5$ [Color figure can be viewed at wileyonlinelibrary.com]

compared to the pure cellulose solutions. Both neat cellulose solutions (L0C8, L0C11.4) exhibit their maximum critical draw ratio at about 10,000 Pas in zero-shear viscosity, but solutions containing lignin all peak at or below 4000 Pas. Consequently, as the viscosity decreases from 10,000 Pas, the critical draw ratio for the lignin solutions continues to increase, while a decrease in critical draw ratio is evident for pure cellulose solutions. From our data, we can conclude that the addition of lignin to a cellulose solution appears to increase the 
maximum critical draw ratio, which also occurs at comparatively lower viscosities.

In our previous publication we suggested that the reduction of critical draw ratio at higher temperatures is due to draw resonance, which causes the rupture of the filament. ${ }^{17}$ Thus, if this is the case, the pure cellulose solutions suffer from draw resonance at higher viscosities compared to the solutions that contain lignin.

The difference between first and last filament breakage was consistently largest for L70C8. This was assumed to be due to inhomogeneities in the solution as a result of the high solid content (26.7 wt\%) which may have led to insufficient dissolution, even though no particles were visible in the micrographs. This observation, in combination with the high temperatures needed for spinning, resulted in that no further experimental investigation of the L70C8 solution was made.

\section{2 | Shear rheology}

Solutions of cellulose in different ILs have previously been extensively studied by shear rheology, in the attempt to explain their spinning behviour. ${ }^{27-30}$ Therefore, the solutions were analyzed with oscillatory shear rheology and the master curves of the complex viscosity for L0C8, L30C8, L50C8, and L0C11.4 are available in Figure 4. Further analysis shows that the viscosity curves for solutions with the same amount of cellulose, L0C8, L30C8, and L50C8, may be shifted to complete overlap. This indicates that addition of lignin affects the overall viscosity in the same way as temperature in the studied frequency range. This means that lignin contributes to the viscosity in a purely viscous manner; either lignin has a relaxation time shorter than the studied frequency range, less than $0.05 \mathrm{~s}$, or that in the prepared solutions the lignin does not align with shear at all. The latter case is plausible, in a recent study no preferred orientation was found in the lignin phase of the fibers. ${ }^{31}$ By increasing the cellulose content, as for solution L0C11.4, the viscosity curve can no longer be shifted to complete overlap, as seen in Figure 4, which shows that a higher cellulose concentration creates more chain entanglements and thereby increases the average relaxation time. The shear modulus of the solutions followed the same trends as viscosity, available in Figure S1, where the addition of lignin did not alter the modulus at the cross over point (COP, $\left.\mathrm{G}^{\prime}=\mathrm{G}^{\prime \prime}\right)$, which is known to be sensitive for molecular weight distribution in solution. ${ }^{32}$ No change of the moduli at the COP is an additional indication that the lignin does not alter the viscoelastic behavior of the solutions. Consequently, the linear shear rheology could not be used to explain the difference observed in spinning behavior.

\section{3 | Extensional rheology}

During air-gap spinning, the solution will predominantly undergo an extensional deformation and an attempt to study the extensional rheology of cellulose solutions was thus made. Three solutions; L0C8, L30C8, and L50C8, were analyzed with Capillary Extension Break-up Rheometry (CaBER). Figure 5 shows the diameter of the solution filament versus time. The filament lifetime, time for filament break-up, increased with the polymer concentration of solution, which have a higher viscosity. ${ }^{33}$

Haward et al. ${ }^{34}$ measured the extensional rheology of cellulose-EMIMAc solutions with Capillary Breakup Extensional Rheometry (CaBER). Slightly before filament
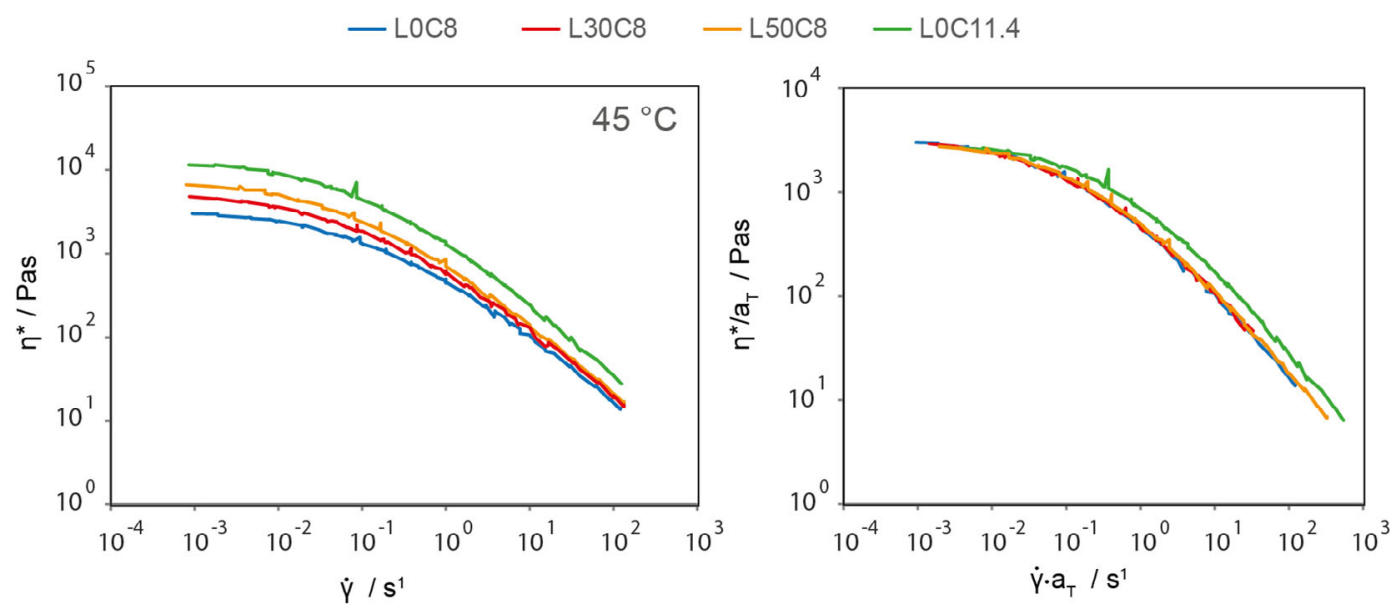

F I G U R E 4 Master curves of complex viscosity for L0C8, L30C8, L50C8, and L0C11.4. In the right plot, the curves have been shifted to overlap according to time-temperature superposition ${ }^{19}$ [Color figure can be viewed at wileyonlinelibrary.com] 


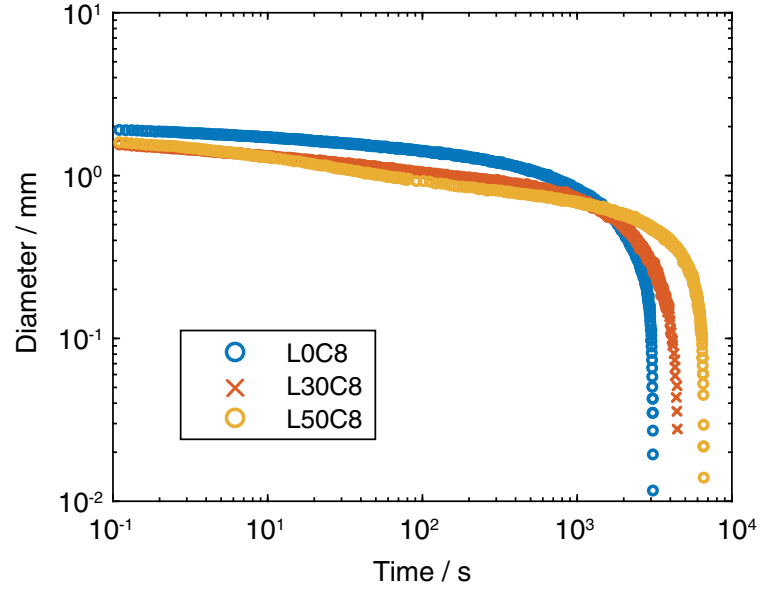

F I G U RE 5 Diameter versus time for solutions with 0\%, 30\%, and $50 \%$ lignin [Color figure can be viewed at wileyonlinelibrary.com]

break-up they identified that the diameter decreased exponentially with time, and from this measured time they could calculate the extensional viscosity. From the results obtained within this work, no exponential decay of the filament diameter was recorded and thus no relaxation time nor extensional viscosity at high Hencky strains could be calculated, and the extensional viscosity of solutions with or without lignin could not be compared. $^{35}$ This is unfortunately a common scenario for concentrated polymer solutions ${ }^{29}$; that is, the decay of the filament diameter is controlled by the viscosity and not the elasticity of the solution. As the polymer concentration increases the viscosity, it may increase more dramatically than the relaxation time. To estimate the relation between viscous and elastic forces during the experiment the elasto-capillary number were calculated for each solution according to Clasen, ${ }^{36}$ and in all cases the elasto-capillary numbers are $<1$ (all values are available in Table S3) which does indicate a viscositycontrolled thinning. This is also in line with the result that the filament lifetime increased with polymer concentration and viscosity.

\section{4 $\mid$ Surface tension}

Another factor in the context of spinnability is the surface tension of the extended solution, which is often neglected, but becomes increasingly important relative to the elastic forces of extended polymer chains in solutions of lower viscosity, that is, solutions at high temperature. ${ }^{37}$ The surface tension of the studied solutions seems to be related to the polymer concentration, see Figure 6 . The reduced surface tension with polymer concentration

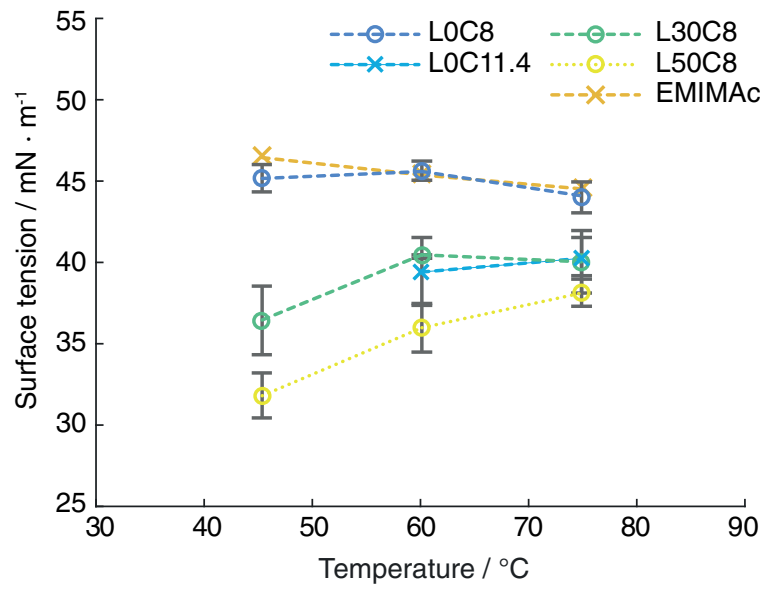

F I G U RE 6 Surface tension of solutions measured with pendant drop [Color figure can be viewed at wileyonlinelibrary.com]

indicates that the cellulose and lignin are surface active. Lignin moieties with hydrophilic functionalities such as carboxylate groups may well be surface active, due to the hydrophobicity of the aromatic ring. ${ }^{38}$ There are numerous cellulose derivatives that are known to be surface active, ${ }^{39}$ however in this study the cellulose is underivatized and the results are not as easily explained. The increase in surface tension with temperature implies a lowered polymer concentration at the solution surface, ${ }^{40}$ which perhaps is a result of better solvability of cellulose and lignin in EMIMAc at higher temperatures. The results follow the same trends as were obtained by Schuermann et al., ${ }^{25}$ who measured the surface tension of cellulose-EMIMAc solutions. A lower surface tension results in a reduction of the retractive forces during filament extension, thus aiding formation of fine filaments. ${ }^{41}$ However, since lignin and cellulose seem to have similar effect on the surface tension of the solution, surface tension was not considered a primary reason for an increased critical draw ratio of solutions that contained lignin.

\section{5 | Stress in filaments during spinning}

The take-up force was measured to investigate the stress build-up with draw ratio for the different solutions, and to get an indication of the breaking stress of solution. Data were collected for draw ratio 1 up to the critical draw ratio. The stress will depend on the viscosity of the solution as well as on the stored elastic stress. As the solutions are shear thinning, it is reasonable to assume that the viscosity will decrease with increased draw ratio. Elasticity is a memory effect; chains that are extended 
during the stretching want to move back into their original state. The faster the fluid is deformed, that is, at higher draw ratio, more and more chains will not have time to relax into their original shape. The elastic stress will thus increase until all chains are at their maximum elongated state. In Figure 7, the stress is plotted for solutions spun at a temperature lower than the temperature of their maximum critical draw ratio, see Figure 2. As can be observed, the stress increases up to a critical draw ratio for all solutions. This data confirms that at temperatures lower than the temperature for maximum critical draw ratio; the filaments undergo a cohesive breakage and they break once the breaking stress is reached. However, from these data it is difficult to extract a breaking stress since the measured force becomes invalid as soon as some filaments start to break.

In Figure 8, the stress is plotted for solutions spun at or above the temperature of their maximum critical draw ratio, see Figure 2. Compared to Figure 7, all stress curves exhibit a different behavior, in this case a maximum stress is noted for all solutions, but less clear for L0C11.4. It is notable that fiber breakage does not occur at the maximum stress, which indicates another breaking mechanism than cohesive breakage. Such mechanism may be necking and/or draw resonance which causes a locally reduced cross section and thus stress amplification leading to filament break-up. In our previous study it was concluded that for a solution with 50:50 lignin: cellulose, draw resonance was observed for temperatures above the temperature at the maximum critical draw ratio. ${ }^{17}$ It can be assumed that a pure cellulose solution would suffer from a similar disturbance at warmer temperatures.

A maximum in the stress - draw ratio curve could be interpreted as the point of an overall chain-chain

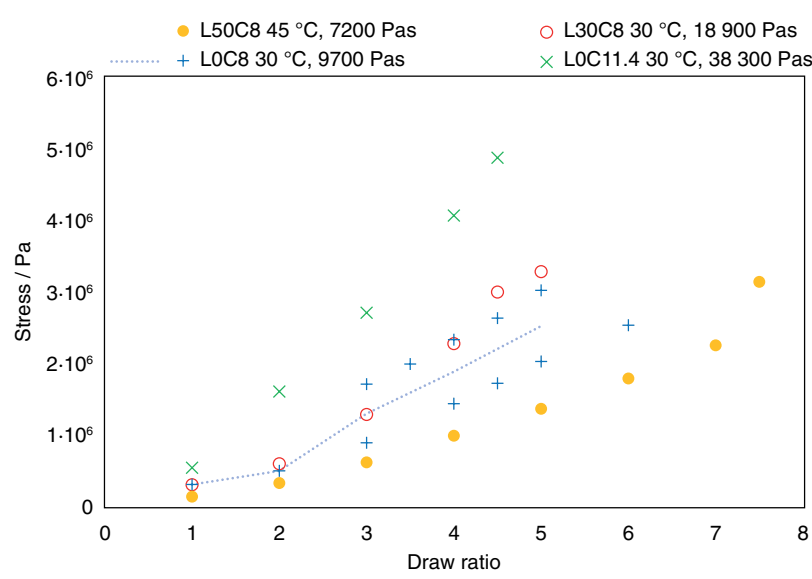

F I G U RE 7 Calculated stress in filaments during spinning at lower temperatures. The dotted line is an average of the L0C8 data, added to guide the eye [Color figure can be viewed at wileyonlinelibrary.com] slippage, which eventually also will cause filament breakage. The stress curves for the solutions containing lignin, L50C8 and L30C8, are different from L0C8. Some sort of a shoulder, more pronounced for L50C8, in the stress curve can be seen between 2 and 4 in draw ratio. Instead of turning downward as the L0C8 stress curve does, the L50C8 and L30C8 curves continues to increase. All three solutions have the same cellulose content and around draw ratio 3 all the cellulose chains may approach their maximum extensibility. Indeed, previous studies have found that the mechanical properties of pure cellulose fibers reaches a plateau at comparable draw ratios, ${ }^{15,42}$ indicating that for pure cellulose fibers, an extended structure is achieved already at low draw ratios. In some way it seems that lignin introduces other interactions in the solution that enables an increase in elasticity and/or viscosity which causes the total stress to increase; all in all that hinders cellulose chain slippage and thereby stabilizes spinning at higher draw ratios. One of the main differences between lignin and cellulose is the difference in molecular weight, Kraft lignin has much lower molecular weight average than cellulose dissolving pulp. ${ }^{12,43}$ The beneficial effect on spinnability of mixing molecular weights in solution has been shown previously and may be contributing also in the current study. ${ }^{44,45}$ Another notable difference between lignin and cellulose is their structure both regarding morphology and molecular structure. The addition of lignin to the solution may introduce additional hydrophobic interactions due to the aromaticity of lignin. In a theoretical study based on mathematical modeling, the preferred adsorption of lignin on cellulose was found to be a stacking of the lignin phenyl rings parallel to the pyranose rings in cellulose,

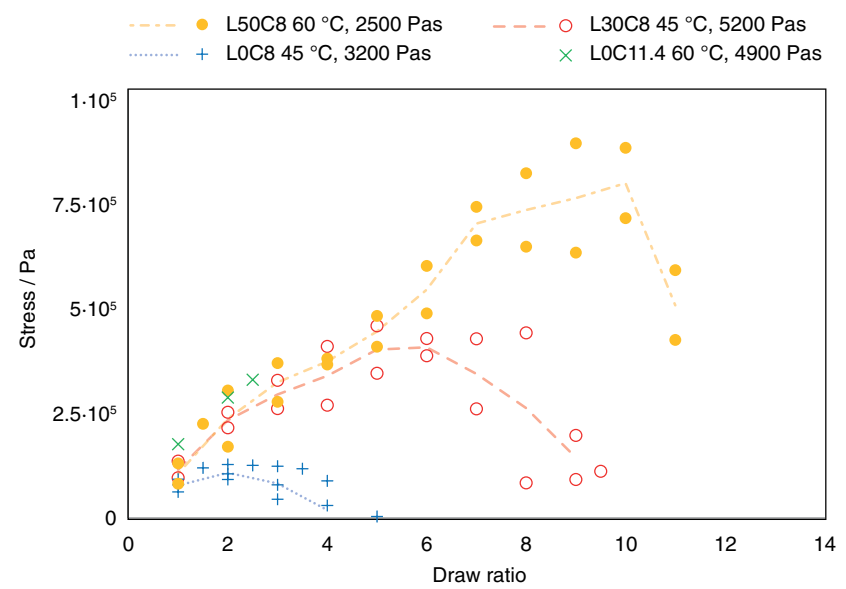

F I G U RE 8 Calculated stress in filaments during spinning at higher temperatures. The lines are averages of their corresponding solution, added to guide the eye [Color figure can be viewed at wileyonlinelibrary.com] 




FI G URE 9 Tensile strength and tensile modulus versus draw ratio for $\mathrm{L} 50 \mathrm{C} 8$ ( $50 \%$ lignin) at $60^{\circ} \mathrm{C}$ [Color figure can be viewed at wileyonlinelibrary.com]

indicating hydrophobic interactions between the two components. ${ }^{46}$

\section{6 | Mechanical properties}

To motivate a high draw ratio during spinning it is necessary to analyze the impact of draw ratio on mechanical properties. That draw ratio can be used to improve the tensile strength and modulus of cellulose fibers is known, ${ }^{15}$ however it is not obvious that this effect remains for fibers consisting of 50\% lignin since lignin is a non-linear and amorphous polymer. As can be seen in Figure 9 the mechanical properties do improve with draw ratio also for such fibers. SEM-images of the fibers are available in Figure S8. The tenacity of the fibers somewhat stagnates after a draw ratio of 8 , but the modulus increases over the whole possible range of draw ratios. The mechanic properties confirm the result in Figure 8, that the structure in a lignin: cellulose fiber is evolving beyond the draw ratio where the corresponding cellulose fiber is reaching a maximum extendable state. Consequently, it is highly motivated to perform spinning at a temperature corresponding to the maximum critical draw ratio to optimize fiber properties. A high degree of preferred orientation in the precursor is also necessary to achieve carbon fibers with high tensile modulus. ${ }^{1}$ To further improve the mechanical properties for example the effect of spinneret geometry and choice of coagulant remains to be investigated.

\section{4 | CONCLUSIONS}

It was found that the addition of lignin increased the maximal critical draw ratio of a cellulose solution. The difference observed in spinning behavior could not be explained neither by shear rheology nor surface tension of the solutions. Measurements of the take-up force on the other hand, demonstrated beneficial effects of adding lignin, as the stress in filaments spun at higher temperatures could be increased. A possible explanation is that lignin has the ability to hinder cellulose-cellulose slippage and thereby improve the spinning stability. From the mechanical properties of filaments with 50:50 lignin: cellulose spun with different draw ratios, it was possible to conclude that both the strength and, in particular, the modulus of the lignin-cellulose fibers increased with a higher draw ratio. The results show the benefit of a high critical draw ratio and motivates to perform spinning at the temperature of maximum critical draw ratio.

\section{ACKNOWLEDGMENTS}

This work was partly performed within the project LightFibre, a collaboration between RISE, Chalmers University of Technology, The Royal Institute of Technology, Valmet AB and SCA Forest Products AB, financed by the Swedish Energy Agency.

\section{ORCID}

Jenny Bengtsson (1) https://orcid.org/0000-0002-2513-4289 Kerstin Jedvert (1) https://orcid.org/0000-0003-2893-8554 Tobias Köhnke (1D) https://orcid.org/0000-0003-1259-6414 Hans Theliander (1) https://orcid.org/0000-0002-21206513

\section{REFERENCES}

[1] E. Frank, L. M. Steudle, D. Ingildeev, J. M. Sprl, M. R. Buchmeiser, Angew. Chem. Int. Ed. 2014, 53, 5262.

[2] Zindani, D.; Maity, S. R.; Bhowmik, S. AIP Conf. Proc. AIP Publishing LLC, 2018.

[3] N. Williams Portal, K. Lundgren, H. Wallbaum, K. Malaga, J. Mater. Civ. Eng. 2015, 27, 14207.

[4] M. Raugei, D. Morrey, A. Hutchinson, P. Winfield, J. Clean. Prod. 2015, 108, 1168.

[5] H. Mainka, O. Täger, E. Körner, L. Hilfert, S. Busse, F. T. Edelmann, A. S. Herrmann, J. Mater. Res. Technol. 2015, 4, 283.

[6] P. Morgan, Carbon Fibers and Their Composites, CRC Press, Boca Raton, FL 2005, p. 791.

[7] A. Bengtsson, P. Hecht, J. Sommertune, M. Ek, M. Sedin, E. Sjöholm, ACS Sustainable Chem. Eng. 2020, 8, 6826.

[8] Garoff, N.; Protz, R.; Erdmann, J.; Ganster, J.; Lehmann, A. A process for the manufacture of a shaped body 2017.

[9] G. Gellerstedt, Ind. Crop Prod. 2015, 77, 845.

[10] A. Bengtsson, J. Bengtsson, C. Olsson, M. Sedin, K. Jedvert, H. Theliander, E. Sjöholm, Holzforschung 2018, 72, 1007.

[11] N. Byrne, R. De Silva, Y. Ma, H. Sixta, M. Hummel, Cellulose 2018, 25, 723.

[12] C. Olsson, E. Sjöholm, A. Reimann, Holzforschung 2017, $71,275$.

[13] T.-F. Yeh, T. Yamada, E. Capanema, H.-M. Chang, V. Chiang, J. F. Kadla, J. Argi. Food Chem. 2005, 53, 3328. 
[14] Y. Ma, S. Asaadi, L. S. Johansson, P. Ahvenainen, M. Reza, M. Alekhina, L. Rautkari, A. Michud, L. Hauru, M. Hummel, H. Sixta, ChemSusChem 2015, 8, 4030.

[15] H. Sixta, A. Michud, L. Hauru, S. Asaadi, Y. Ma, A. W. T. King, I. Kilpeläinen, M. Hummel, Y. Ma, C. Cross, E. J. Bevan, Beadle C 2015, 30, 43.

[16] B. Wirth, M. Warnecke, B. Schmenk, G. Seide, T. Gries, Chem. Fibers Int. 2011, 61, 64.

[17] J. Bengtsson, K. Jedvert, T. Köhnke, H. Theliander, J. Appl. Polym. Sci. 2019, 136, 47800.

[18] Wallmo, H. Lignin extraction from black liquor: precipitation, filtration and washing; Chalmers University of Technology, 2008.

[19] T. G. Metzger, The Rheology Handbook, Vincentz Network, Hanover 2014, p. 135.

[20] C. Macosko, Theology: Principles, Measurments and Applications, Wiley-VCH, New York, NY 1994, p. 109.

[21] W. P. Cox, E. H. Merz, J. Polym. Sci. A 1958, 28, 619.

[22] A. Ziabicki, Fundamentals of Fibre Formation, Wiley, Bath 1976, p. 64.

[23] A. Ziabicki, Fundamentals of Fibre Formation, Wiley, Bath 1976, p. 274.

[24] R. Sescousse, K. A. Le, M. E. Ries, T. Budtova, J. Phys. Chem. B 2010, 114, 7222.

[25] J. Schuermann, T. Huber, D. LeCorre, G. Mortha, M. Sellier, B. Duchemin, M. P. Staiger, Cellulose 2016, 23, 1043.

[26] H. F. D. Almeida, A. R. R. Teles, J. A. Lopes-da-Silva, M. G. Freire, J. A. P. Coutinho, J. Chem. Thermodyn. 2012, 54, 49.

[27] B. Kosan, C. Michels, F. Meister, Cellulose 2008, 15, 59.

[28] R. J. Sammons, J. R. Collier, T. G. Rials, S. Petrovan, J. Appl. Polym. Sci. 2008, 110, 1175.

[29] M. Hummel, A. Michud, S. Asaadi, Y. Ma, L. Hauru, E. Hartikainen, H. Sixta, Annu. Trans. Nord. Rheol. Soc. 2015, 23,13 .

[30] L. K. J. Hauru, M. Hummel, K. Nieminen, A. Michud, H. Sixta, Soft Matter 2016, 12, 1487.

[31] L. Svenningsson, J. Bengtsson, K. Jedvert, W. Schlemmer, H. Theliander, L. Evenäs, Carbohydr. Polym. 2021, 254, 117293.

[32] F. Meister, B. Kosan, Nord. Pulp Pap. Res. J. 2015, 30, 112.

[33] O. Arnolds, H. Buggisch, D. Sachsenheimer, N. Willenbacher, O. Arnolds, H. Buggisch, D. Sachsenheimer, N. Willenbacher, Rheol. Acta 2010, 49, 1207.
[34] S. J. Haward, V. Sharma, C. P. Butts, G. H. McKinley, S. S. Rahatekar, Biomacromolecules 2012, 13, 1688.

[35] S. L. Anna, G. H. Mckinley, Cit. J. Rheol. 2001, 45, 115.

[36] Clasen, C. Capillary breakup extensional rheometry of semidilute polymer solutions; 2010; 22.

[37] M. Bechert, B. Scheid, Phys. Rev. Fluids 2017, 2, 113905.

[38] N. Alwadani, P. Fatehi, Carbon Resour. Convers. 2018, 1, 126.

[39] L. Karlsson, in Surfactants from Renewable Resources (Eds: I. Johansson, M. Kjellin), John Wiley \& Sons, Chichester, UK 2010, p. 253.

[40] G. J. Fleer, M. A. C. Stuart, J. M. H. M. Scheutjens, T. Cosgrove, B. Vincent, Polymers at Interfaces, Springer, Dordrecht 1998, p. 396.

[41] W. Stibal, R. Schwarz, U. Kemp, K. Bender, F. Weger, M. Stein, Ullmann's Encycl. Ind. Chem. Wiley-VCH Verlag, Weinheim 2005.

[42] S. Elsayed, M. Hummel, D. Sawada, C. Guizani, M. Rissanen, H. Sixta, Cellulose 2021, 28, 533.

[43] D. Klemm, B. Philipp, T. Heinze, U. Heinze, W. Wagenknecht, Comprehensive Cellulose Chemistry, Volume 1: Fundamentals and Analytical Methods, Wiley-VCH, Weinheim 1998, p. 9.

[44] A. Michud, M. Hummel, H. Sixta, Polymer 2015, 75, 1.

[45] L. Palangetic, N. K. Reddy, S. Srinivasan, R. E. Cohen, G. H. Mckinley, C. Clasen, Polymer 2014, 55, 4920.

[46] C. J. Houtman, R. H. Atalla, Plant Physiol. 1995, 107, 977.

\section{SUPPORTING INFORMATION}

Additional supporting information may be found online in the Supporting Information section at the end of this article.

How to cite this article: Bengtsson J, Jedvert $\mathrm{K}$, Köhnke T, Theliander H. The challenge of predicting spinnability: Investigating benefits of adding lignin to cellulose solutions in air-gap spinning. J Appl Polym Sci. 2021;e50629. https:// doi.org/10.1002/app.50629 